\title{
Medición de la Capacidad Emprendedora de ingresantes a la Facultad de Ingeniería Industrial de la UNMSM
}

\begin{abstract}
RESUMEN
El propósito de la investigación fue determinar la capacidad de emprendimiento de los alumnos que ingresaron a la Facultad de Ingeniería Industrial del año 2008 utilizando la de Habilidad Emprendedora, validada por Flores (UIGV, 2003) Asimismo, se analizaron los factores que marcan diferencias en esta capacidad, tales como el tipo de preparación para acceder a la universidad, el género o la procedencia de colegio de secundaria. Los resultados indican que los alumnos sujetos de estudio, de acuerdo al instrumento utilizado presentan una habilidad emprendedora alta.
\end{abstract}

Palabras clave: Habilidad emprendedora, Emprendimiento.

MEASUREMENT OF THE ENTREPRENEURS OF ENTRANTS TO THE FACULTY OF ENGINEERING INDUSTRIAL OF THE UNIVERSIDAD NACIONAL MAYOR DE SAN MARcos

\section{ABSTRACT}

The purpose of the investigation was to determine the ability of students who venture entered the $\mathrm{Fa}$ culty of Engineering in 2008 using the entrepreneurial skills, validated by Flores (UIGV, 2003). We also analyzed the factors that make difference in this capacity, such as the type of preparation for university, gender, or source of secondary school. The results suggest that students study subjects, according to the instrument used, have a high entrepreneurial skill

Key words: Entrepreneurial Skills, Entrepreneurship.

\section{INTRODUCCIÓN}

La capacidad de emprendimiento es un tema que concita la atención tanto de países europeos como sudamericanos y, en el caso peruano, de instituciones públicas (Ministerios, Universidades, entre otros) como privadas (ADEX, Universidades, ONGs). Coinciden todos ellos en que la capacidad emprendedora es un aspecto importante para el desarrollo de las sociedades.

Según la organización Global Entrepreneurship Monitor, entidad que estudia y registra la capacidad emprendedora, "para que una economía pueda competir y crecer con éxito es necesario equilibrar la reserva de empresas fomentando su creación y también administrar su transmisión. Algunos estudios sugieren que existe una correlación positiva entre espíritu empresarial y crecimiento. [...] Un crecimiento sostenible basado en la innovación y la excelencia exige un número creciente de nuevas empresas, que puedan proporcionar más y mejores empleos".

El Estado peruano, a través de diferentes entidades, entre ellas la Dirección Regional de Comercio y Turismo (DIRCETUR), promueve constantemente jornadas de sensibilización y/o capacitación respecto a la promoción de un cultura emprendedora, sobre todo en el marco de los tratados de libre comercio suscritos con diversos países, entre ellos EEUU.

El ex Ministro de Trabajo, Fernando Villarán desarrolló el 2001 una investigación sobre las competencias necesarias para la creación y gestión exitosa de pequeñas y microempresas, destacando la necesidad de fomentar una cultura de innovación y emprendimiento.

Portocarrero (2003) llevó a cabo una investigación sobre la capacidad de emprendimiento en estudiantes de Administración y Psicología de la UNFV, determinando que las estudiantes de Administración presentaban una mayor predisposición a generar sus propios negocios.

Un estudio respecto a la capacidad de emprendimiento de estudiantes de Europa y América Latina fue presentado por Rusque (1998) al XII Congreso Internacional de Espíritus Emprendedores, celebrado en San José de Costa Rica, en él se menciona que la "capacidad emprendedora se manifiesta mediante la concepción de un proyecto que contenga elementos innovadores y el grado en que se logra materializarlo, es decir, de transformar, cambiar de forma, de idea a realidades en un plazo y con recursos definidos. El emprendedor, que se identifica por su

Ingeniero Industrial. Docente de la UNMSM y UNFV. E-mail: otinocog@unmsm.edu.pe 
capacidad emprendedora, concibe proyectos innovadores y viables, los materializa y establece las condiciones que aseguren la sobrevivencia y el mejoramiento de su idea".

En la facultad de Ingeniería Industrial (UNMSM), los docentes investigadores Inche, Quispe y Sandoval (1998) desarrollaron un estudio sobre la implementación de incubadoras de negocios agroindustriales con alcance a todo el teritorio nacional. Mientras que Garcia (2001) establecia en las conclusiones de una investigacion respecto a la relacion universidad y las PYMES, la necesidad de implementar semilleros empresariales con el concurso de estudiantes, egresados y docentes.

\section{El espíritu emprendedor}

Un concepto de bastante aceptación sobre el espíritu emprendedor es el siguiente: "habilidad de la persona para transformar las ideas en actos. Está relacionado con la creatividad, la innovación y la asunción de riesgos, así como con la habilidad para planificar y gestionar proyectos con el fin de alcanzar objetivos".

Como resultado de esta concepción la Unión Europea considera el fomento del espíritu emprendedor como uno de los objetivos cruciales en sus políticas de empleo, educación y económicas.

En el mismo documento, haciendo un balance del proceso de formación profesional en universidades españolas se constata que "cuando se desarrollan actividades encaminadas a la generación de actividades emprendedoras, el enfoque suele ser de carácter más técnico (cómo crear una empresa) que centrado en el desarrollo de las capacidades necesarias (cómo fomentar la cultura emprendedora como parte de las competencias y valores de la persona).

Serida (2008) describe a un emprendedor como "aquella persona que aplica su talento creativo para iniciar su propia empresa o engrandecer una ya existente y es capaz de generar un ambiente favorable para el incremento de las oportunidades de negocio y grado de innovación en las organizaciones y por ende contribuir con el desarrollo de su entorno". En seguida añade, respecto a los emprendedores en el ámbito universitario, que "la formación emprendedora genera grandes beneficios para el estudiante debido a que no solo desarrolla habilidades de gestión empresarial sino complementa el desarrollo profesional del alumno desarrollando o fortaleciendo competencias emprendedoras tales como liderazgo, trabajo en equipo, motivación, comunicación, creatividad, planificación, entre otros que le permitirán generar emprendimientos exitosos, mejorar su calidad de vida y la de otros".

\section{Universidad y Emprendimiento}

Diferentes universidades peruanas, estatales y privadas, han puesto especial atención al fomento del emprendimiento entre los estudiantes. La Universidad Nacional Mayor de San Marcos (UNMSM) se encuentra entre ellas con una serie de acciones, como son talleres de formación y convocatoria a jóvenes emprendedores. Tal es el caso de la Jornada de Emprendedorismo (Setiembre 2008) organizado por el Vicerrectorado de Investigación

La Universidad Peruana de Ciencias (UPC), a través de su Instituto INVERTIR, impulsa el Programa Líder Acción, con la participación de 200 jóvenes del interior del país.

Por su parte, la Pontificia Universidad Católica del Perú ha creado el Centro de Innovación y Desarrollo Emprendedor, mediante el cual desarrolla el Programa Diseño e Innovación en la empresa; entidad que realiza periódicamente convocatorias públicas para canalizar experiencias de esta índole a nivel de Lima Metropolitana.

En el caso de la Universidad del Pacífico, se promueve el Programa de Capacitación "Emprendedores dinámicos".

\section{La capacidad de emprendimiento de los ingresantes 2008 en la facultad de Ingeniería Industrial}

Las universidades forman profesionales para atender la demanda laboral especializada en diferentes aspectos de la actividad económica del país en su conjunto. La UNMSM forma, entre otros profesionales orientados a la acción empresarial, ingenieros industriales, en donde se les proporciona un conjunto de herramientas orientados al manejo de empresas; sin embargo, su plan de estudios no aborda el tema del emprendimiento de una manera explícita y sistemática.

\section{MATERIALES Y MÉTODOS}

El propósito de la presente investigación fue determinar la capacidad de emprendimiento de los ingresantes a la Facultad de Ingeniería Industrial del año 2008. En primera instancia se abordó la capacidad emprendedora de los ingresantes y luego se dis- 
cernió sobre los factores que marcan diferencias en esta capacidad, tales como el tipo de preparación para acceder a la universidad, el género o la procedencia de colegio de secundaria.

Se aplicó un cuestionario a 132 alumnos 2008 de un total de 170 , los cuales fueron seleccionados aleatoriamente.

\begin{tabular}{|c|c|c|c|}
\hline \multicolumn{4}{|c|}{ Cuadro N 01: Población y Muestra } \\
\hline Género & Población & $\%$ & Muestra \\
\hline Varones & 117 & $69 \%$ & 91 \\
\hline Mujeres & 53 & $31 \%$ & 41 \\
\hline Total & 170 & $100 \%$ & 132 \\
\hline FUENTE: elaboración propia en base a información FII \\
\hline \multicolumn{4}{|l}{} \\
\hline
\end{tabular}

Se aplicó la Escala de Habilidad Emprendedora, validada por Flores (UIGV, 2003), la misma que cuenta con cinco áreas: conocimiento de sí mismo y auto confianza, visión de futuro, motivación de logro, planificación y persuasión.

La calificación de esta escala contempla para cada una de las áreas:

\begin{tabular}{|l|l|}
\hline \multicolumn{2}{|c|}{$\begin{array}{r}\text { Cuadro } \\
\text { N 02: Escala de Calificación } \\
\text { del instrumento }\end{array}$} \\
\hline Puntaje & Calificación \\
\hline De 1 a 4 & Muy bajo \\
\hline De 5 a 8 & Bajo \\
\hline De 9 a 12 & Mediano \\
\hline De 13 a 16 & Alto \\
\hline De 17 a 20 & Muy alto \\
\hline FuENTE: elaboración propia \\
\hline
\end{tabular}

\section{RESULTADOS}

El cuadro $N^{\circ} 03$ revela que los ingresantes 2008 presentan una alta habilidad emprendedora en todas las áreas evaluadas (la calificación se basa en el cuadro $\mathrm{N}^{\circ}$ 02), con un comportamiento ho- mogéneo en todas ellas, dado que el coeficiente de variación es menor a $20 \%$. En lo que respecta al conocimiento de sí mismo se observa un comportamiento mucho más homogéneo que en las otras dimensiones (14\% de coeficiente de variación).

Según el cuadro 04, hay diferencias en el promedio obtenido por los ingresantes, según su colegio de procedencia. Se observa que el mayor promedio lo obtienen los de colegios privados (79.94) con una mayor homogeneidad que el de los otros segmentos $(12.80 \%)$.

Aplicando la prueba ANOVA (cuadro $N^{\circ}$ 05) para comparar las medias de los tres tipos de colegios se obtuvieron los siguientes resultados, según los cuales la diferencia observada es estadísticamente significativa (valor $\mathrm{p}$ menor que 0.05).

Otro factor considerado, respecto a la capacidad emprendedora de los alumnos ingresantes 2008, fue el tipo de preparación pre universitaria de los mismos.

Conforme al cuadro $\mathrm{N}^{\circ} 06$ el mejor promedio respecto al puntaje total corresponde a los ingresantes que tuvieron como principal fuente de preparación para el ingreso a la UNMSM su colegio pre universitario $(78,71)$. Si bien todos los tipos de preparación registran un comportamiento homogéneo (coeficiente de variación menor que $20 \%$ ), el más homogéneo corresponde a aquellos que se prepararon en colegio pre universitario.

Al comparar los promedios del puntaje total teniendo como factor el tipo de preparación pre universitaria se obtuvo para la prueba ANOVA correspondiente (ver cuadro $\mathrm{N}^{\circ}$ 07).

De acuerdo a estos resultados, la diferencia entre los promedios es estadísticamente significativa.

Considerando el género como factor de diferenciación de los puntajes en la escala de habilidad emprendedora, se obtuvieron los siguientes resultados (ver cuadro $\mathrm{N}^{\circ}$ 08).

\begin{tabular}{|c|c|c|c|c|}
\hline \multicolumn{5}{|c|}{ Cuadro $N^{\circ}$ 03: Puntaje total obtenido según área de evaluación } \\
\hline Area & Media & Desviación típica & $\begin{array}{l}\text { Coeficiente } \\
\text { Variación }\end{array}$ & Calificación \\
\hline Conocimiento de sí mismo & 72,18 & 10,42 & $14 \%$ & Alto \\
\hline Visión de futuro & 14,48 & 2,56 & $18 \%$ & Alto \\
\hline Motivación de logro & 14,22 & 2,39 & $16 \%$ & Alto \\
\hline Planificación & 15,2 & 2,49 & $17 \%$ & Alto \\
\hline Persuasión & 13,86 & 2,54 & $18 \%$ & Alto \\
\hline
\end{tabular}




\begin{tabular}{|c|c|c|c|c|}
\hline \multicolumn{5}{|c|}{ Cuadro N. 04: Puntaje Total según institución educativa $^{-}$} \\
\hline I.E. de procedencia & $\mathrm{n}$ & Media & $\mathrm{S}$ & CV \\
\hline Estatal & 73 & 70,26 & 10,61 & $15,11 \%$ \\
\hline Privada & 50 & 74,94 & 9,59 & $12,80 \%$ \\
\hline Parroquial & 9 & 72,44 & 10,97 & $15,14 \%$ \\
\hline Total & 132 & 72,18 & 10,42 & \\
\hline
\end{tabular}

FUENTE: Elaboración propia

\begin{tabular}{|c|c|c|c|c|c|}
\hline \multicolumn{6}{|c|}{ Cuadro N. ${ }^{\circ}$ 05: Prueba ANOVA: Factor instituciones educativas. Puntaje Total } \\
\hline & $\begin{array}{c}\text { Suma de } \\
\text { cuadrados }\end{array}$ & gl & Media cuadrática & F & Sig. \\
\hline Intergrupos & 650,539 & 2 & 325,270 & 3,090 &, 049 \\
\hline Intragrupos & 13581,097 & 129 & 105,280 & & \\
\hline Total & 14231,636 & 131 & & & \\
\hline
\end{tabular}

FUENTE: Elaboración propia

\begin{tabular}{|l|c|c|c|c|}
\hline \multicolumn{5}{|c|}{ Cuadro N. ${ }^{\circ}$ 06: Puntaje Total según tipo de preparación } \\
\hline Preparación Pre Universitaria & $\mathrm{n}$ & Media & $\mathrm{S}$ & $\mathrm{CV}$ \\
\hline Centro Pre UNMSM & 49 & 69,12 & 10,02 & $14,50 \%$ \\
\hline Academia Privada & 68 & 73,47 & 10,7 & $14,56 \%$ \\
\hline Colegio Preuniversitario & 15 & 78,71 & 8,83 & $11,22 \%$ \\
\hline Total & 132 & 72,18 & 10,42 & \\
\hline
\end{tabular}

FUENTE: Elaboración propia

\begin{tabular}{|l|c|c|c|c|c|}
\hline \multicolumn{5}{|c|}{ Cuadro N. $^{\circ}$ 07: Prueba ANOVA: Factor preparación pre universitaria. Puntaje Total } \\
\hline & Suma de cuadrados & gl & Media cuadrática & F & Sig. \\
\hline Intergrupos & 904,501 & 3 & 301,500 & 2,896 &, 038 \\
\hline Intragrupos & 13327,135 & 128 & 104,118 & & \\
\hline Total & 14231,636 & 131 & & & \\
\hline
\end{tabular}

FUENTE: Elaboración propia

\begin{tabular}{|c|c|c|c|c|}
\hline \multicolumn{5}{|c|}{ Cuadro N. $^{\circ}$ 08: Prueba t de Student: Factor género } \\
\hline & Sexo & N & Media & Desviación típ. \\
\hline Puntaje Total & Masculino & 91 & 72,44 & 11,124 \\
\hline & Femenino & 41 & 71,61 & 8,769 \\
\hline
\end{tabular}

FUENTE: Elaboración propia

Se observa que los promedios según género son ligeramente diferentes, pero el valor $p$ obtenido en la prueba t de Student (0.674) denota que dicha diferencia no es estadísticamente significativa.

\section{CONCLUSIONES}

1. Los ingresantes 2008 a Ingeniería Industrial, de acuerdo a la escala validada por Flores (UIGV, 2003), presentan una habilidad emprendedora alta, tanto en el puntaje total como en cada una de las áreas.
2. El colegio de procedencia y el tipo principal de preparación preuniversitaria son factores de diferenciación en la habilidad emprendedora

3. El género no constituye un factor de diferenciación respecto a la habilidad emprendedora.

\section{REFERENCIAS BIBLIOGRÁFICAS}

1. BID (2002). Empresarialidad en Economías Emergentes: la creación de nuevas empresas en América Latina y Asia del Este. Development Bank of Japan, Institute National University Of General Sarmiento. 
2. Colectivo Integral de Desarrollo (2008). Programa emprendedores dinámicos. http://www.cid.org.pe/HomePage/index.htm (Visitado el 2008 - 10 - 30)

3. Comunidad Europea de Naciones (2004). Guía de buenas prácticas para promover las actitudes y capacidades empresariales mediante la educación.

4. Comunidad Europea de Naciones (2006). "Fomentar la mentalidad empresarial mediante la eduación y la formación".

5. Flores, Pedro (2003). Capacidad emprendedora en estudiantes ingresantes a la Universidad Inca Garcilazo de la Vega. Tesis de Maestría.

6. García, Teonila (2001). La universidad y su compromiso con las PYMES. Industrial Data 4 (2): 11-17.

7. Global Entrepreneurship Monitor (2004). Espíritu emprendedor y crecimiento. http://www.banespyme.org/Banespymes. do?id=15\&prefix=/publicaciones\&page=/toFicha.do (Visitado el 2008-07-15).

8. Inche, Jorge; Sandoval, César y Quispe, Carlos (1998). Implementación de incubadora de negocios agroindustriales en la Facultad de Ingeniería Industrial de la UNMSM. Revista Industrial DATA. 1 (1): 9-16.

9. Pontificia Universidad Católica del Perú (2008). Centro de Innovación y Desarrollo Empresarial. http://www.pucp.edu.pe/cide/ (Visitado el 2008 $10-30)$.

10. Portocarrero, Carlos (2003). Capacidad emprendedora y calificación empresarial en estudiantes de Administración y Psicologia de la UNFV. Tesis de Maestria UNFV. Lima.

11. Rusque, A. (1998). Medición de la capacidad emprendedora de estudiantes de Europa y América Latina. Ponencia XII Congreso Internacional de Emprendedores. San José de Costa Rica.

12. Villarán, Fernando (2001). Creación y gestión exitosa de PYMES. Universidad del Pacífico. Lima.

13. Unión Europea Informe (2006). Fomentar la mentalidad empresarial" http://www.ipyme.org/IPYME/es/UnionEuropea/UnionEuropea/PoliticaEuropea/ProyectosBest/FomentoEspirituEmprendedor.htm (Visitado el 2008 - 07 - 15).

14. Universidad de Ciencias Aplicadas (2008). Instituto Invertir. http://www.upc.edu.pe (Visitado el 2008 - 10 - 30)

15. Universidad del Pacífico (2008). Programa emprendedores dinámicos. http://www.up.edu.pe (Vi-sitado el 2008 - 10 - 30) 


\section{Anexo}

\section{ESCALA DE HABILIDAD EMPRENDEDORA}

Estimado alumno, el siguiente cuestionario es parte de una investigación orientada a la generación de negocios como parte del quehacer del Ingeniero Industrial. Te agradecemos por la valiosa colaboración que prestas a este estudio, y esperamos llenes el documento con objetividad.

En las siguientes afirmaciones debes marcar una, y solo una, de las siguientes opciones.

\begin{tabular}{|c|c|c|c|c|c|}
\hline 0 & 1 & 2 & 3 & 4 & 5 \\
\hline Nunca & Casi nunca & En ocasiones & Con frecuencia & Casi siempre & Siempre \\
\hline
\end{tabular}

1. Identifica sus talentos y los usa para alcanzar sus propósitos

2. Actúa por iniciativa propia usando sus recursos

3. Identifica, calcula y controla los riesgos al emprender las acciones

4. Atribuye a sí mismo las causas y consecuencias de sus acciones

5. Propone nuevas alternativas para alcanzar sus propósitos

6. Visualiza anticipadamente el resultado de sus acciones

7. Da soluciones fluidas $y / 0$ flexibles frente a los problemas

8. Busca y toma oportunidades para resolver sus demandas o exigencias

9. Busca perfeccionarse y superarse como persona

10.Mantiene vitalidad para desarrollar las actividades

11. Cumple con los compromisos adquiridos

12.Aplica controles de calidad (hace bien las cosas)

13. Define metas o propósitos concretos en su desempeño

14. Investiga, explora, curiosea, pregunta

15.Administra racionalmente los recursos

16. Evalúa y corrige las acciones

17. Comprende y satisface las necesidades del interlocutor

18. Trabaja cooperativamente en equipo

19. Influye en los demás

20.Construye redes de apoyo

¡Muchas gracias!

\section{INFORMACIÓN GENERAL}

A. Edad: (años cumplidos)

B. Sexo: (1) Masculino (2) Femenino

C. La Institución Educativa donde terminó la educación secundaria fue:
(1) Estatal
(2) Privada
(3) Parroquial

D. Su preparación pre universitaria principal para ingresar a la UNMSM la realizó en:
(1) Estudió por su cuenta
(2) Estudió en el Centro Pre Universitario de la UNMSM
(3) Academia Pre Universitaria Privada
(4) Colegio Pre Universitario
(5) Otrros:

E. En qué año terminó la educación secundaria:
(1) 2007
(2) 2006
(3) 2005
(4) 2004 o antes 